\title{
Analysis and Prognosis of Tumor Immune Infiltrates and Immune Microenvironment of m6A-Related LncRNAs in Patients with Gastric Cancer
}

Jiarong Huang

North Sichuan Medical University

Jinxuan Song

North Sichuan Medical University

Xiangyu Li

North Sichuan Medical University

Shuangfei Liu

North Sichuan Medical University

Wentao Huang

North Sichuan Medical University

Ziyi Shen

North Sichuan Medical University

Yan Cheng

North Sichuan Medical University

Shien Kou

North Sichuan Medical University

\section{Zhenguo Gao}

The Affiliated Nanchong Central Hospital of North Sichuan Medical College (University)

Yunhong Tian ( $\nabla$ drtianyunhong@126.com )

The Affiliated Nanchong Central Hospital of North Sichuan Medical College (University)

Jiani Hu

Wayne State University

\section{Research Article}

Keywords: m6A-Related IncRNA, Immune Infiltrates, Immune microenvironment, Gastric cancer

Posted Date: November 3rd, 2021

DOl: https://doi.org/10.21203/rs.3.rs-987733/v1 
License: (c) (i) This work is licensed under a Creative Commons Attribution 4.0 International License. Read Full License 


\section{Abstract \\ Background}

Studies have shown that long noncoding RNAs and N6-methyladenosine play an important role in gastric cancer. The purpose of this study was to determine the correlation and prognostic value of m6A-related IncRNAs and immune infiltration in gastric cancer.

\section{Methods}

We downloaded the clinically related information and RNA-Seq transcriptome data of gastric cancer patients from the TCGA database. Univariate Cox regression analysis and Pearson analysis were used to screen out m6A-related IncRNAs. Consensus cluster analysis was used to divide the sample into two clusters, and LASSO analysis and minimum absolute shrinkage were used to construct a risk scoring model.

\section{Results}

A total of 25 IncRNA expression profiles were screened, and gastric cancer patients were divided into different subtypes. Cluster 2 had a better prognosis, but its matrix score, estimate score and immune score were low. Cluster 1 was rich in resting memory CD4 T cells, regulatory T cells, monocytes, and resting mast cells, and Cluster 2 was rich in activated memory CD 4 T cells and follicular helper T cells. Thirteen IncRNAs were selected to construct a risk model, and the prognosis of gastric cancer patients in the high-risk group was poor. The expression of PD-L1 in tumors is significantly higher than that in normal tissues. Univariate and multivariate Cox regression analysis results showed that the overall survival rate was significantly related to stage and risk score, which can be used as an independent prognostic factor. The results of the heat map and scatter plot showed that clusters $(P=0.0045)$ and grade $(G 1-2, G 3, P=0.0037)$ were significantly related to prognosis. The results of the relationship between the risk score and immune cell infiltration showed that memory B cells, resting dendritic cells, MO macrophages, and M2 macrophages were positively correlated with risk scores, while resting mast cells, monocytes, activated NK cells, and follicular helper T cells were negatively correlated with risk scores.

\section{Conclusion}

The results of this study indicate that m6A-related IncRNAs may play an important role in the prognosis of gastric cancer patients and the tumor immune microenvironment and provide help for the treatment of gastric cancer patients.

\section{Background}


In recent years, with the advancement of medical standards and the popularization of health concepts, the global morbidity and mortality of gastric cancer have declined, but it is still a major public health problem ${ }^{[1]}$. Gastric cancer is the fourth most common cancer and the second leading cause of cancer death in the world ${ }^{[2]}$, with approximately 738,000 deaths every year ${ }^{[3]}$. As a common RNA modification, m6A exists in IncRNAs and microRNAs and plays an important role in regulating the splicing and translation of IncRNAs ${ }^{[4-6]}$. In recent years, many studies have shown that the tumor microenvironment plays an important role in cancer progression ${ }^{[7-10]}$. Studies have shown that m6A modification is closely related to the tumor microenvironment and PD-L1 expression in hepatocellular carcinoma and cholangiocarcinoma ${ }^{[11-14]}$, and the prognostic model of m6A-related IncRNAs can be used to predict the overall survival of patients with various tumors ${ }^{[15-20]}$, but the clinical application and immunotherapy effect of m6A-related IncRNAs in the prognosis of gastric cancer are still unclear.

This study aims to evaluate the correlation of m6A-related IncRNAs with immune cell infiltration and the prognosis of gastric cancer patients. By constructing a gastric cancer prognostic model, they were divided into high-risk groups and low-risk groups to determine whether m6A-related IncRNAs could be used as prognostic biomarkers for gastric cancer.

\section{Materials And Methods}

\section{Data collection}

The clinically related information and IncRNA expression data of gastric cancer patients were downloaded from the TCGA database. The clinically related information mainly included sex, age, classification, grade, and TNM staging. Continuous variables were converted to categorical variables, and the chi-square test was used to compare the variables in the training cohort and validation cohort.

\section{Identification of m6A-related genes and prognosis-related m6A IncRNAs}

We extracted the expression matrix of m6A-related genes based on the mRNA expression data in the TCGA database, used the "Limma R" software package to filter m6A-related IncRNAs $(P<0.05$, Cor $>0.5)$, and then visualized them as a coexpression network graph. Using univariate Cox regression analysis with $\mathrm{P}<0.05$ as the screening standard, m6A-related prognostic IncRNAs were screened out. The Wilcoxon statistical method was used to detect the differential expression of IncRNAs between gastric cancer tissues and normal tissues.

\section{Consensus clustering identifies m6A-related IncRNA subgroups}

We used the "Consensus cluster plus" software package to cluster gastric cancer patients into different subtypes to explore the biological characteristics of m6A-related IncRNAs. Kaplan-Meier survival 
analysis and log-rank tests were used to analyze the differences in clinicopathological factors between the two groups.

The correlation between different clusters and time.

The "ESTIMATE" software package was used to calculate matrix, estimate and immune scores, and Wilcoxon was used to test the differential expression of matrix, estimate and immune scores between the two clusters. The cibersort algorithm was used to evaluate the immune cell type score of each sample. The Wilcoxon test was used to show the abundance of immune infiltrating cells between the two clusters.

\section{Construction and validation of the risk model and its relationship with clinicopathological characteristics and immune cell infiltration}

The least absolute contraction and LASSO regression algorithm were used to screen the m6A-related IncRNAs that are most closely related to overall survival. Then, a risk model was built. The formula for the risk score was coef $1 * x 1+\operatorname{coef} 2^{\star} \times 2+\operatorname{coef} 3 * x 3+\ldots+\operatorname{coefi}{ }^{*} x i$ (coef refers to the coefficient of each IncRNA, and $X$ refers to the expression level of IncRNA). According to the median risk score, patients were divided into high-risk groups and low-risk groups. Kaplan-Meier survival analysis was used to detect the difference in overall survival between the high- and low-risk groups. According to clinicopathological characteristics, subgroup analysis, univariate Cox regression analysis and multivariate Cox regression analysis were performed to evaluate whether the risk score can be used as an independent prognostic factor. Pearson correlation tests were used to evaluate the relationship between the risk score and immune cell infiltration.

\section{Results}

\section{Identification of m6 related IncRNA in gastric cancer (GC) patients}

We extracted the expression of IncRNAs and m6A-related genes from the TCGA database and used Pearson's correlation value $>0.5$ and $\mathrm{P}<0.01$ as the criteria for screening m6A-related IncRNAs. The coexpression network of m6A-related IncRNAs is shown in Figure 1A. Through univariate Cox regression analysis, 25 IncRNAs were obtained $(P<0.05)$. The forest plot results showed that 12 IncRNAs were risk factors $(H R>1)$, and the remaining 13 IncRNAs were protective factors $(H R<1)$ (Figure 1B). The heat map and box plot show the expression of 25 IncRNAs in gastric cancer tissues and normal tissues (Figure 1C, Figure 1D).

\section{m6A-related IncRNA consensus clustering}


Using Consensus Clustering to cluster the samples, the CDF curve shows that when $\mathrm{k}=2$, the interference between subgroups is the smallest, and the difference is significant (Figure 2A-B). According to the relative change of the area under the CDF curve and the tracking plot, the k value was determined to be 2 to 9 (Figure 2C-D). The survival chart results show that patients in the Cluster 2 subgroup had a higher overall survival rate (Figure $2 \mathrm{E}, \mathrm{P}<0.05$ ).

\section{Immune cell infiltration and tumor immune microenvironment (TIME) and genetic correlation analysis}

We analyzed the scores of 22 immune cell types, and the results showed that Cluster 1 was rich in resting memory CD $4 T$ cells, regulatory T cells, monocytes, and resting mast cells (all $P<0.05$ ). Cluster 2 was rich in activated memory CD4 T cells and follicular helper $T$ cells (Figure $3 A$, all $P<0.05$ ). To further understand the relationship between m6A-related IncRNAs and immunity, the estimate algorithm was used to calculate the distribution difference of the immunity ratio. Cluster 1 had a higher estimate score, immune score and stromal score (Figure 3B-D). The correlation between m6A-related IncRNAs showed that there was a positive correlation between these IncRNAs (Figure 3E). The expression of PD-L1 in the two clusters was not significantly different (Figure 3F). The expression of PD-L1 in tumors was significantly higher than that in normal tissues (Figure 3G).

\section{Construction and verification of a prognostic model based on m6A-related IncRNAs}

We used LASSO regression analysis to identify prognostic-related m6A IncRNAs (Figure 4A-B). Based on the median risk score, we divided gastric cancer patients into high-risk groups and low-risk groups. The survival analysis results showed gastric cancer in the high-risk group. The patient's survival rate is poor (Figure 4C-D). The risk score and survival status distribution results of each patient in the training cohort and the validation cohort show that the higher the risk score, the higher the patient's mortality rate, and the significantly reduced survival time. The heat map shows that the expression patterns of 13 IncRNAs in the high- and low-risk groups were different (Figure 4E-F). The above results indicate that the risk score based on 13 m6A-related IncRNAs has high predictive power for the prognosis of gastric cancer patients.

\section{Prognostic risk score is related to clinicopathological characteristics and immune cell infiltration}

We used univariate and multivariate Cox regression analyses to determine whether a risk model based on m6A-related IncRNAs can be used as an independent prognostic factor for predicting gastric cancer patients. Univariate Cox regression analysis showed that the overall survival rate was significantly related to stage and risk score. Stage $(H R=1.866, P<0.001)$ and risk score $(H R=1.587, P<0.001)$ in the training group were independent prognostic factors. The results of multivariate Cox regression analysis showed that age $(H R=1.027, P<0.032)$ and stage $(H R=1.412, P<0.024)$ in the test group were independent prognostic factors. Stage $(H R=2.015, P<0.001)$ and risk score $(H R=1.666, P<0.001)$ in the train group were independent prognostic factors (Figure 5A-D). 
We further evaluated the relationship between risk and the prognosis of 13 m6A-related IncRNAs. The results of the heat map and scatter plot showed that clusters $(P=0.0045)$ and grade $(G 1-2, G 3, P=0.0037)$ were significantly related to prognosis. (Figure $6 \mathrm{~A}-\mathrm{K}$ ). We further explored the relationship between the risk score and immune cell infiltration (Figure $7 \mathrm{~A}-\mathrm{H})$, and the results showed that memory $B$ cells, resting dendritic cells, M0 macrophages, and M2 macrophages were positively correlated with risk scores (all P $<0.01$ ), while resting mast cells, monocytes, activated NK cells, and follicular helper T cells were negatively correlated with risk scores (all $\mathrm{P}<0.01$ ).

\section{Discussion}

Characterized by the low early diagnosis rate, high malignancy and poor prognosis, GC is a serious threat to human health in our country. At present, the malignant progression of GC has not been fully elucidated. Determining new biomarkers for the diagnosis and prognosis of $\mathrm{GC}$ has become a prerequisite to conquer GC. Accumulating evidence indicates the significant influences of IncRNAs on tumorigenesis. IncRNAs impact multiple mixed dimensions in the occurrence and progression of tumors, especially at the transcriptional and posttranscriptional levels ${ }^{[26]}$. For example, Ning Cui experimentally observed ${ }^{[27]}$ that LINC00511 acted as a therapeutic target in GC treatment, targeting inducing the expression of STAT3 by inhibiting the expression of miR-625-5p. LINC00649 functions as an oncogenic IncRNA in accelerating cell proliferation, migration and epithelial-mesenchymal transition ${ }^{[28]}$. With the continuous development of molecular biotechnology, IncRNAs related to GC have been discovered one after another, but they are still in rudimentary stages. As the most prevalent internal modification of RNA, m6A has been thoroughly and widely studied recently. Published reports indicated that m6A modification targeted at IncRNAs could affect both the occurrence and progression of GC. Four m6A-methylated and expressed IncRNAs were identified, including RASAL2-AS1, LINC00910, SNHG7 and LINC01105, which exerted regulatory roles on GC cell proliferation ${ }^{[29]}$. However, the prognostic significance and tumor immune mechanism of m6Arelated IncRNAs remain elusive.

This study analyzed m6A-related IncRNAs related to the clinical characteristics of gastric cancer patients and found that 12 IncRNAs were risk factors $(H R>1)$ and 13 IncRNAs were protective factors $(H R<1)$. The risk score constructed from 13 m6A-related IncRNAs was also significantly related to overall survival, clusters and grade $(\mathrm{G} 1-2, \mathrm{G} 3)$ were significantly related to prognosis, Cluster 1 was related to the high-risk group, and its prognosis was poor. This shows that the risk model based on m6A-related IncRNAs is reliable.

We constructed a risk score model including thirteen IncRNAs, such as LINC00106, TYMSOS, MED8-AS1, SREBF2-AS1, AL390961.2, AC144546.1, and AC005586.1.

Wang and his colleagues found that the expression of LINC00106 in thyroid cancer was significantly lower than that in normal tissues. LINC00106 suppressed the metastasis and invasion of cancer cells by inhibiting epigenetic-mesogenic transition as a tumor suppressor ${ }^{[30]}$. A risk score model was developed based on five IncRNAs: LINC00205, TRHDE-AS1, OVAAL, LINC00106, and MIR100HG. Wu verified that 
the ${ }^{[31]}$ incRNA-based risk model performs well in predicting GC prognosis. LINC00106 could be regarded as a significant prognostic biomarker in gastric cancer ${ }^{[32]}$, which reached a consensus with our study. Studies have found ${ }^{[33]}$ that TYMSOS is overexpressed in GC cells and exerts growth-promoting effects on GC. Moreover, TYMSOS, as a competitive endogenous RNA, modulated GC progression at the posttranscriptional level. Unlike the abovementioned investigations, IncRNAs AL390961.2, AC144546.1, and AC005586.1 were first reported in GC, leaving a wide scope for further research.

A number of studies in recent years have shown that m6A can play an important role in cancer immunity through different regulatory factors ${ }^{[21-24]}$. The $\mathrm{m} 6 \mathrm{~A}$ regulatory factor in colon cancer has three $\mathrm{m} 6 \mathrm{~A}$ modification modes, and these three m6A modification modes are closely related to the immunophenotype ${ }^{[25]}$. The tumor immune microenvironment plays an important role in immunotherapy. It has been reported that tumor infiltrating lymphocytes in the tumor microenvironment promote disease progression and increase the chance of invasion and metastasis. M2 macrophage infiltration correlates with a poor prognosis in colon cancer ${ }^{[34]}$. Furthermore, a higher fraction of $\mathrm{M} 0$ macrophages $(P=0.001)$ and a lower fraction of $M 2$ macrophages $(P=0.018)$ were found to be risk factors for a poorer histological grade of hepatocellular carcinoma ${ }^{[35]}$. Some studies have reported that tumor-associated macrophage infiltration is associated with invasion, angiogenesis, and poor prognosis in $\mathrm{GC}^{[36]}$. This study found that Cluster 2 is rich in activated memory CD 4 T cells and follicular helper T cells and has low activation in stromal cells. The results of the relationship between the risk score and immune cell infiltration showed that B cell memory, resting dendritic cells, M0 macrophages, and M2 macrophages were positively correlated with the risk score (all $\mathrm{P}<0.01$ ), while resting mast cells, monocytes, activated NK cells, and follicular helper $\mathrm{T}$ cells were negatively correlated with the risk score (both $\mathrm{P}<0.01$ ). In summary, we analyzed stromal cells and immune infiltrating cells in the tumor immune microenvironment, and the results showed that m6A-related IncRNAs were involved in the reprogramming of the tumor immune microenvironment.

This study still has some limitations. First, the effectiveness of this model still needs to be verified in a large number of external queues. Second, the m6A-related IncRNAs selected in this study need to be verified by further functional experiments. It is necessary to further reveal the regulatory network between m6A and IncRNAs.

In conclusion, this study conducted a bioinformatics study on the regulatory mechanism of m6A-related IncRNAs in gastric cancer and screened 25 m6A-related IncRNAs. Different gastric cancer patients have different IncRNA subtypes in terms of overall survival, and the overall survival of Cluster 2 is higher. The prognostic risk score of m6A-related IncRNAs is closely related to clinicopathological characteristics (grade), two subtypes and immune cell infiltration.

\section{Abbreviations}




\begin{tabular}{|ll|}
\hline Full name & Abbreviations \\
\hline gastric cancer & GC \\
\hline cumulative distribution functions & CDFs \\
\hline tumor immune microenvironment & TIME \\
\hline
\end{tabular}

\section{Declarations}

\section{Ethics approval and consent to participate}

The research article was approved by the ethics committee of Nanchong Central Hospital and was carried out in accordance with the 1975 Helsinki Declaration, Because of the anonymous nature of the data and the opt-out option disclosed on our institution's homepage, the requirement for additional informed consent to participate in this study was deemed unnecessary.

\section{Consent for publication}

Not applicable

\section{Availability of data and materials}

The datasets generated and analyzed during the current study are available in the TCGA repository, [https://www.cancer.gov/about-nci/organization/ccg/research/structural-genomics/tcga]

\section{Competing interests}

The authors declare that they have no competing interests.

\section{Funding}

This work was supposed by the Foundation of Health Commission of Sichuan Province [19PJ061], and Cooperative project of Nanchong City with North Sichuan Medical College [20SXQT0321]. The funding body has no role in the design of the study and collection, analysis, and interpretation of data and inwriting the manuscript.

\section{Authors' contributions}

All authors contributed to the study design as well as the collection and analysis of the data. JH, JS, and $\mathrm{XL}$ designed the research; SL, WH and ZS performed the clinical and basic research; JH, JS and YT performed the statistical analyses; YC, SK, and ZG drafted the work; SL and JH substantively revised it. All authors read and approved the final manuscript.

\section{Acknowledgements}




\section{References}

1. Van Cutsem E, Sagaert X, Topal B, et al. Gastric cancer[J]. The Lancet, 2016, 388(10060): 2654-2664.

2. Crew K D, Neugut A I. Epidemiology of gastric cancer[J]. World journal of gastroenterology: WJG, 2006, $12(3): 354$.

3. Sitarz R, Skierucha M, Mielko J, et al. Gastric cancer: epidemiology, prevention, classification, and treatment[J]. Cancer management and research, 2018, 10: 239.

4. Dai F, Wu Y, Lu Y, et al. Crosstalk between RNA m6A modification and non-coding RNA contributes to cancer growth and progression[J]. Molecular Therapy-Nucleic Acids, 2020.

5. Coker H, Wei G, Brockdorff N. m6A modification of non-coding RNA and the control of mammalian gene expression[J]. Biochimica et Biophysica Acta (BBA)-Gene Regulatory Mechanisms, 2019, 1862(3): 310-318.

6. Lan Y, Liu B, Guo H. The Role of M6A Modification in the Regulation of Tumor Related IncRNAs[J]. Molecular Therapy-Nucleic Acids, 2021.

7. Binnewies $\mathrm{M}$, Roberts $\mathrm{E} \mathrm{W}$, Kersten $\mathrm{K}$, et al. Understanding the tumor immune microenvironment (TIME) for effective therapy[J]. Nature medicine, 2018, 24(5): 541-550.

8. Keren $L$, Bosse M, Marquez D, et al. A structured tumor-immune microenvironment in triple negative breast cancer revealed by multiplexed ion beam imaging[J]. Cell, 2018, 174(6): 1373-1387. e19.

9. Taube J M, Klein A, Brahmer J R, et al. Association of PD-1, PD-1 ligands, and other features of the tumor immune microenvironment with response to anti-PD-1 therapy[J]. Clinical cancer research, 2014, 20(19): 5064-5074.

10. Chen S M Y, Krinsky A L, Woolaver R A, et al. Tumor immune microenvironment in head and neck cancers[J]. Molecular carcinogenesis, 2020, 59(7): 766-774.

11. Shen S, Yan J, Zhang Y, et al. N6-methyladenosine (m6A)-mediated messenger RNA signatures and the tumor immune microenvironment can predict the prognosis of hepatocellular carcinoma[J]. Annals of Translational Medicine, 2021, 9(1).

12. Qiu X, Yang S, Wang S, et al. M6A Demethylase ALKBH5 Regulates PD-L1 Expression and Tumor Immunoenvironment in Intrahepatic Cholangiocarcinoma[J]. Cancer Research, 2021.

13. Li R, Yin Y H, Ji X L, et al. Pan-Cancer Prognostic, Immunity, Stemness, and Anticancer Drug Sensitivity Characterization of N6-Methyladenosine RNA Modification Regulators in Human Cancers[J]. Frontiers in Molecular Biosciences, 2021, 8.

14. Li J, Wang W, Zhou Y, et al. m6A regulator-associated modification patterns and immune infiltration of the tumor microenvironment in hepatocarcinoma[J]. Frontiers in Cell and Developmental Biology, 2021, 9: 1679.

15. Wang H, Meng Q, Ma B. Characterization of the Prognostic m6A-Related IncRNA Signature in Gastric Cancer[J]. Frontiers in Oncology, 2021, 11: 927. 
16. Zheng J, Guo J, Cao B, et al. Identification and validation of IncRNAs involved in m6A regulation for patients with ovarian cancer[J]. Cancer Cell International, 2021, 21(1): 1-18.

17. Yu H, Zhang Z. ALKBH5-mediated m6A demethylation of IncRNA RMRP plays an oncogenic role in lung adenocarcinoma[J]. Mammalian Genome, 2021, 32(3): 195-203.

18. Li Z, Li Y, Zhong W, et al. m6A-Related IncRNA to Develop Prognostic Signature and Predict the Immune Landscape in Bladder Cancer[J]. Journal of Oncology, 2021, 2021.

19. Ji F, Lu Y, Chen S, et al. m6A methyltransferase METTL3-mediated IncRNA FOXD2-AS1 promotes the tumorigenesis of cervical cancer[J]. Molecular Therapy-Oncolytics, 2021.

20. Wen S, Wei Y, Zen C, et al. Long non-coding RNA NEAT1 promotes bone metastasis of prostate cancer through N6-methyladenosine[J]. Molecular cancer, 2020, 19(1): 1-18.

21. Xu S, Tang L, Dai G, et al. Expression of m6A regulators correlated with immune microenvironment predicts therapeutic efficacy and prognosis in gliomas[J]. Frontiers in cell and developmental biology, 2020, 8: 1335.

22. Du J, Ji H, Ma S, et al. m6A regulator-mediated methylation modification patterns and characteristics of immunity and stemness in low-grade glioma[J]. Briefings in Bioinformatics, 2021.

23. Fang $\mathrm{J}$, Hu M, Sun $\mathrm{Y}$, et al. Expression profile analysis of m6A RNA methylation regulators indicates they are immune signature associated and can predict survival in kidney renal cell carcinoma[J]. DNA and Cell Biology, 2020, 39(12): 2194-2211.

24. Wu X R, Chen Z, Liu Y, et al. Prognostic signature and immune efficacy of m1A-, m5C-and m6Arelated regulators in cutaneous melanoma[J]. Journal of cellular and molecular medicine, 2021.

25. Chong W, Shang L, Liu J, et al. m6A regulator-based methylation modification patterns characterized by distinct tumor microenvironment immune profiles in colon cancer[J]. Theranostics, 2021, 11(5): 2201.

26. He, R.Z., D.X. Luo and Y.Y. Mo, Emerging roles of IncRNAs in the post-transcriptional regulation in cancer. Genes Dis, 2019. 6(1): p. 6-15.

27. Cui, N., et al., Long non-coding RNA LINC00511 regulates the expression of microRNA-625-5p and activates signal transducers and activators of transcription 3 (STAT3) to accelerate the progression of gastric cancer. Bioengineered, 2021. 12(1): p. 2915-2927.

28. Wang, $H_{\text {., }}$ et al., Long non-coding RNA LINC00649 regulates YES-associated protein 1 (YAP1)/Hippo pathway to accelerate gastric cancer (GC) progression via sequestering miR-16-5p. Bioengineered, 2021. 12(1): p. 1791-1802.

29. Lv, Z., et al., Joint analysis of IncRNA m(6)A methylome and IncRNA/mRNA expression profiles in gastric cancer. Cancer Cell Int, 2020. 20: p. 464.

30. Wang, X.J., et al., LINC00106 prevents against metastasis of thyroid cancer by inhibiting epithelialmesenchymal transition. Eur Rev Med Pharmacol Sci, 2020. 24(19): p. 10015-10021.

31. $\mathrm{Wu}, \mathrm{Y}$., et al., A risk score model with five long non-coding RNAs for predicting prognosis in gastric cancer: an integrated analysis combining TCGA and GEO datasets. PeerJ, 2021. 9: p. e10556. 
32. Qi, M., et al., Integrated analysis of a ceRNA network reveals potential prognostic IncRNAs in gastric cancer. Cancer Med, 2020. 9(5): p. 1798-1817.

33. Gu, Y., et al., TYMSOS drives the proliferation, migration, and invasion of gastric cancer cells by regulating ZNF703 via sponging miR-4739. Cell Biol Int, 2021. 45(8): p. 1710-1719.

34. Bai, R., et al., Pan-cancer analyses demonstrate that ANKRD6 is associated with a poor prognosis and correlates with M2 macrophage infiltration in colon cancer. Chin J Cancer Res, 2021. 33(1): p. 93-102.

35. Zhang, Z., Z. Wang and Y. Huang, Comprehensive Analyses of the Infiltrating Immune Cell Landscape and Its Clinical Significance in Hepatocellular Carcinoma. Int J Gen Med, 2021. 14: p. 4695-4704.

36. Ma, Y.Y., et al., Interaction of coagulation factors and tumor-associated macrophages mediates migration and invasion of gastric cancer. Cancer Sci, 2011. 102(2): p. 336-42.

\section{Figures}
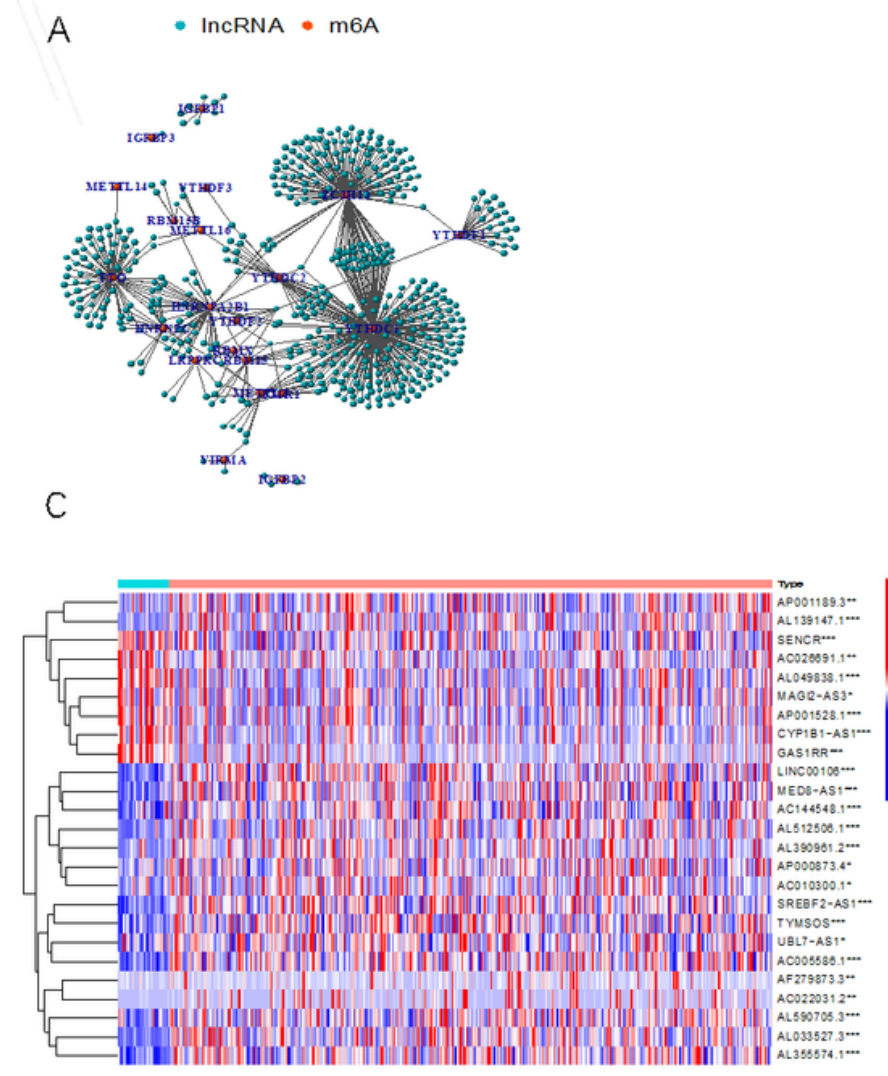

B
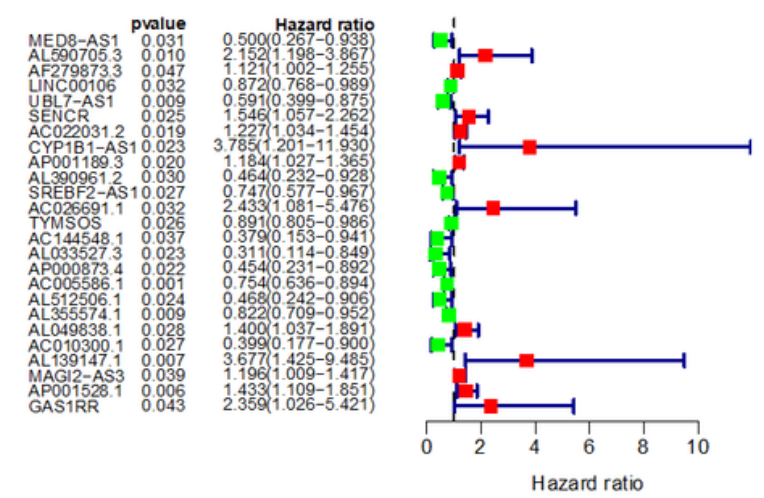

D

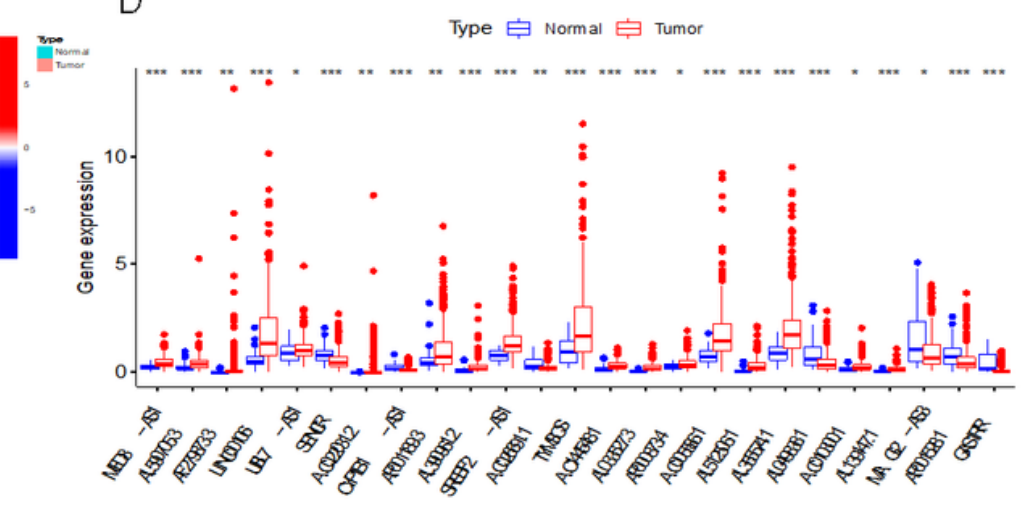

\section{Figure 1}

IncRNAs and m6A-related genes are significantly related (A) m6A-related 


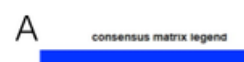
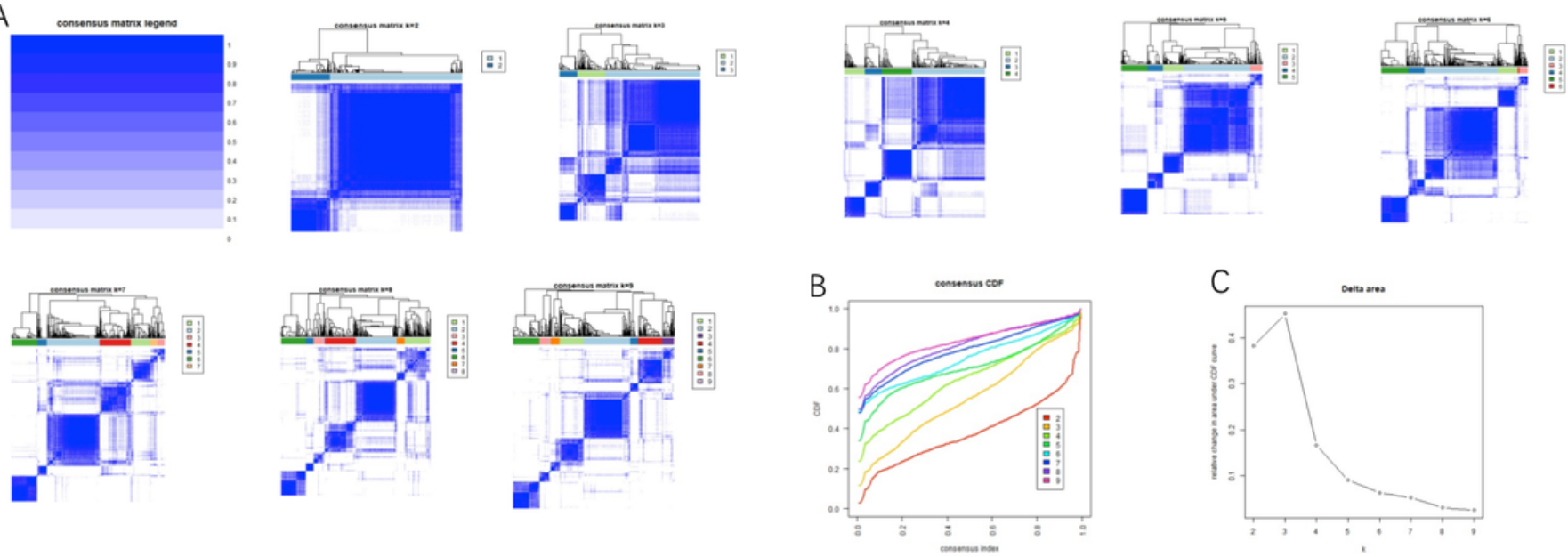

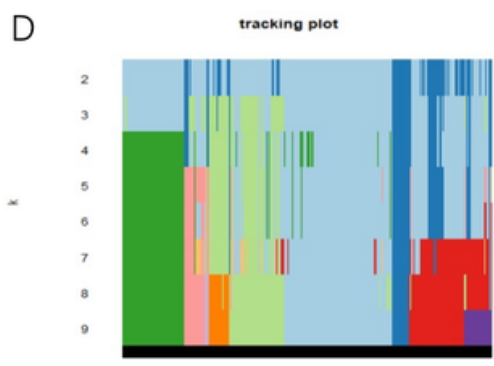

samples
$E$

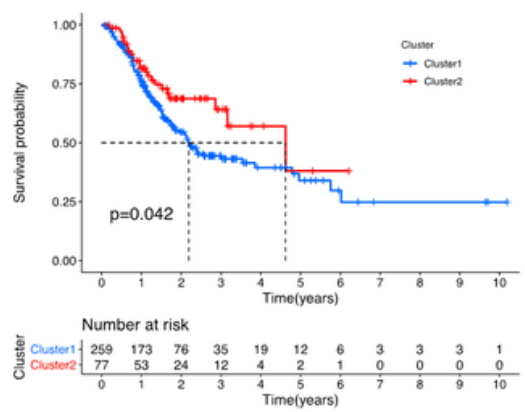

Figure 2

Differential survival outcome of GC in Cluster 1/2 subtypes. (A) Consensus score matrix of all samples when $\mathrm{K}=2, \mathrm{~K}=3, \mathrm{~K}=4, \mathrm{~K}=5, \mathrm{~K}=6, \mathrm{~K}=7, \mathrm{~K}=8$, and $\mathrm{K}=9$. (B) The cumulative distribution functions (CDFs) for $K=2$ to $K=9$. (C)Relative change in area under $C D F$ area for $k=2$ to 9. (D)Tracking plot. (E) Survival analysis of Cluster $1 / 2$ subtypes in the TCGA cohort. 

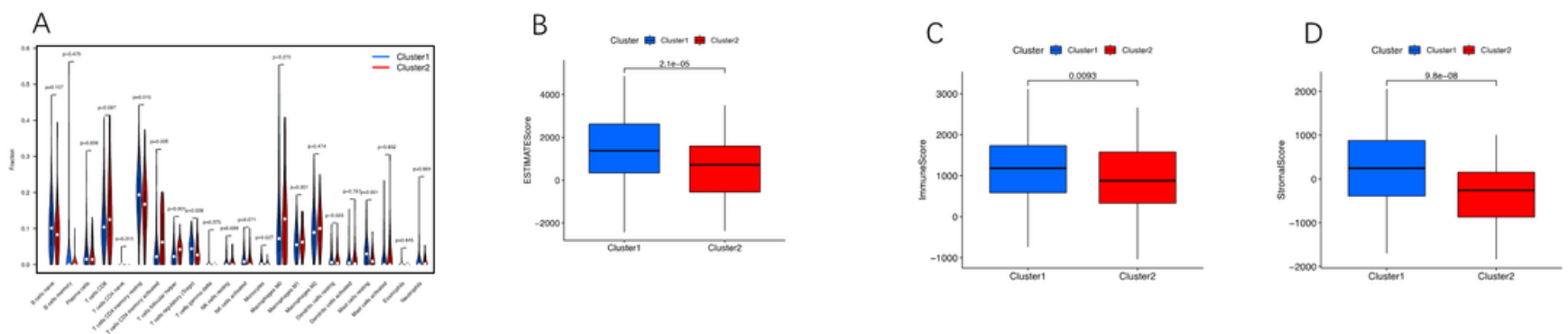

E

$\mathrm{F}$

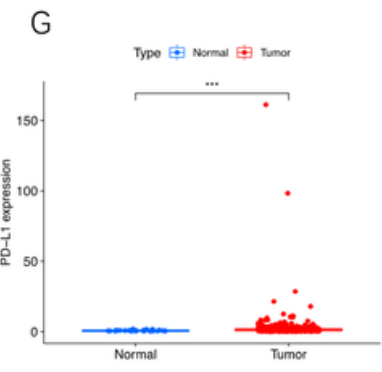

Figure 3

(A) The infiltrating levels of immune cell types in Cluster 1/2 subtypes. (B) Estimate score in two subtypes. (C) Immune score in two subtypes. (D)Stromal score in two subtypes. (E) The correlation between m6A-related IncRNAs and PD-L1 and other genes. (F)PD-L1 expression in two clusters. (G) PD-L1 expression in tumor and normal tissues. 

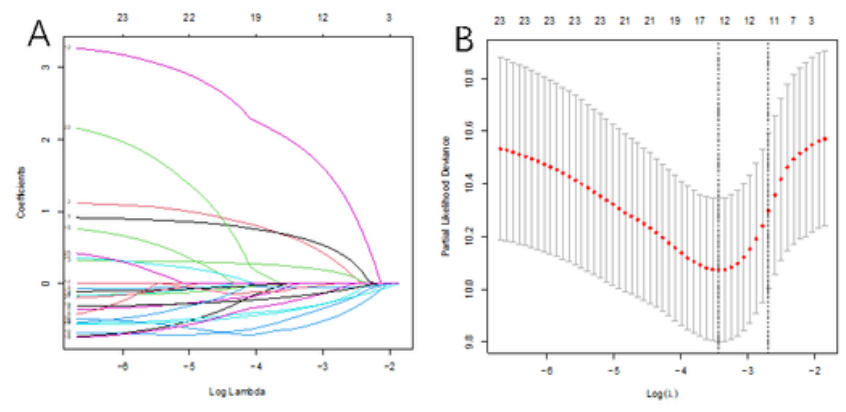

E
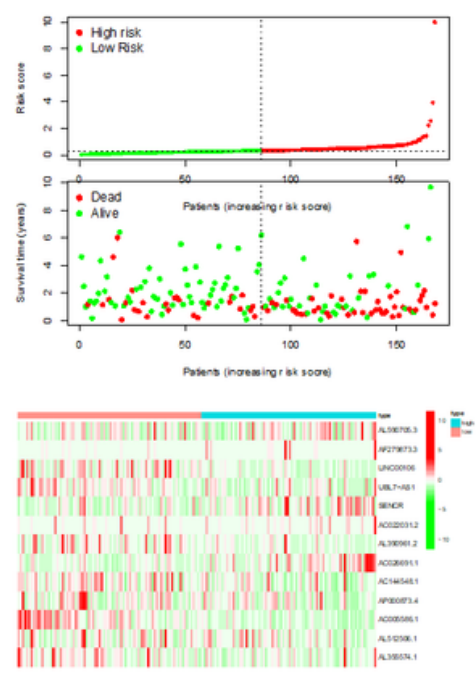

C

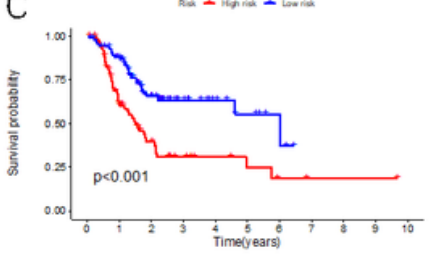

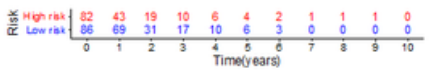

F
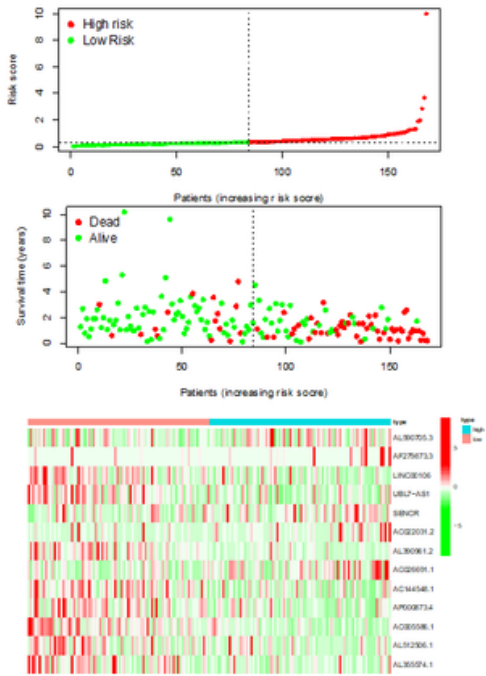

D

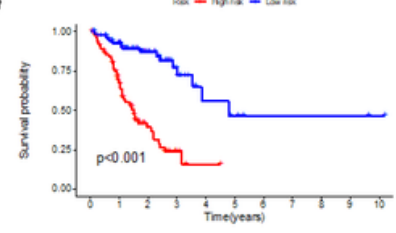

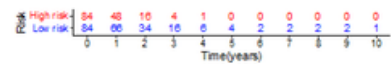

\section{Figure 4}

Construction of a gastric cancer prognostic risk model based on 13 m6A-related IncRNAs. (A-B) Least absolute shrinkage and LASSO regression. (C) Overall survival analysis of high/low-risk patients in the test group. (D) Overall survival analysis of high-/low-risk patients in the training group. (E) Distribution of risk score, survival status and heatmap of the 13 m6A-related IncRNAs in the high/low risk group in the train group. (F) Distribution of risk score, survival status and heatmap of the 13 m6A-related IncRNAs in the high/low risk group in the test group. 
A

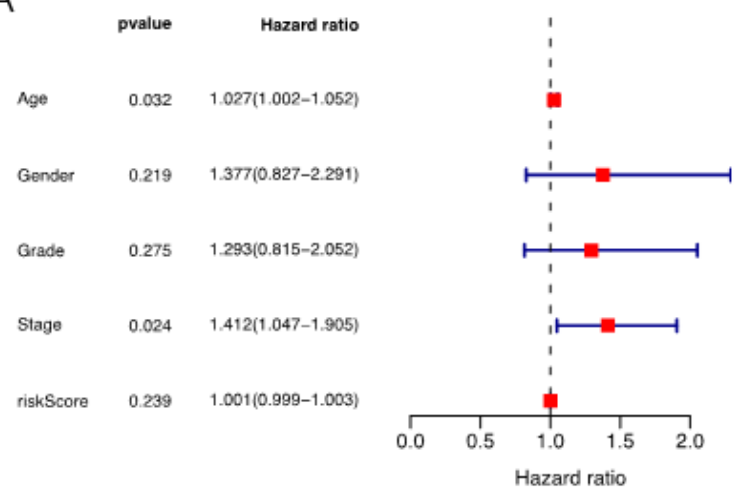

C

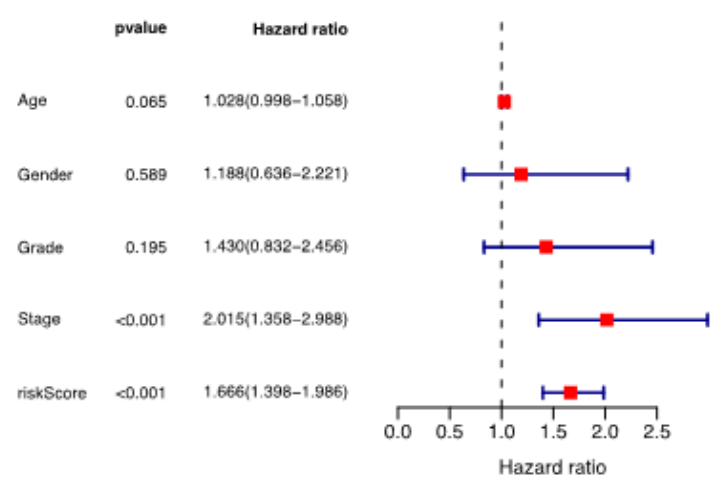

B

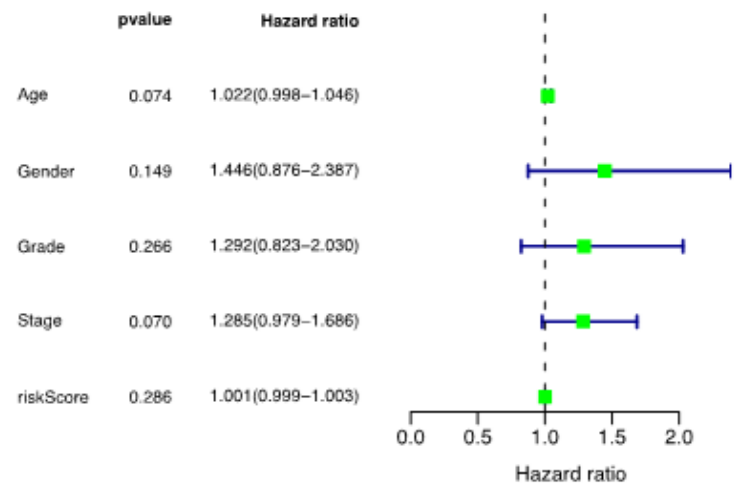

D
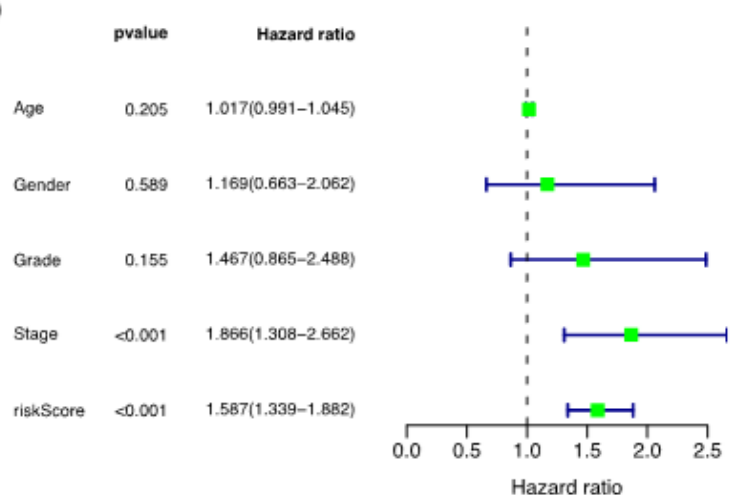

Figure 5

(A) Multiple Cox regression in the test group. (B) Univariate Cox regression in the test group. (C) Multiple Cox regression in the Train group. (D) Univariate Cox regression in the Train group. 

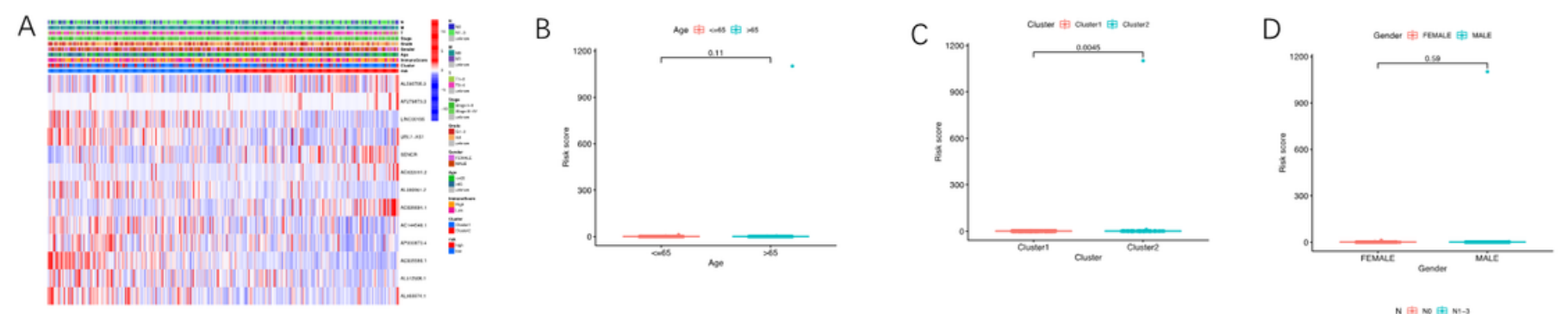

E
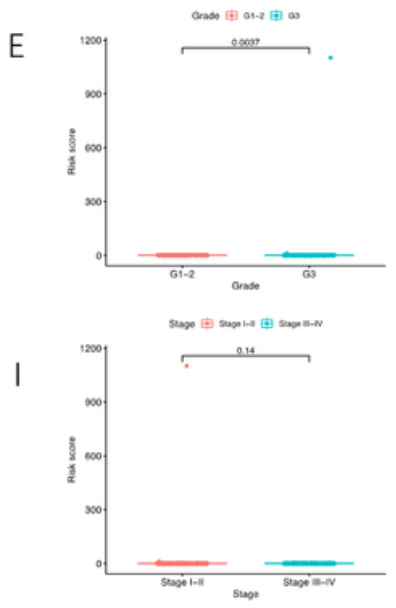

F

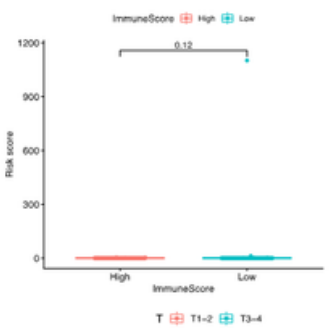

J

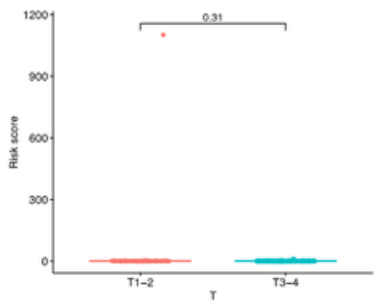

G
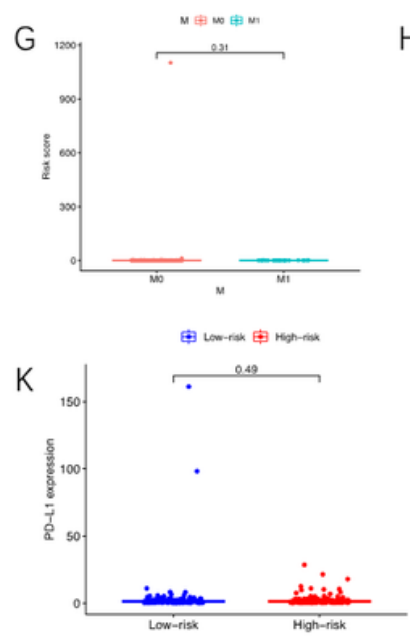

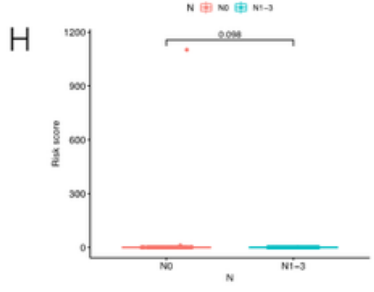

\section{Figure 6}

The prognostic risk score is related to clinicopathological characteristics. (A) Heat maps of clinicopathological characteristics, immune scores and different IncRNA expression patterns in the highand low-risk groups. (B) Distribution of risk scores stratified by age. (C) Distribution of risk scores stratified by Cluster 1/2. (D) Distribution of risk scores stratified by sex. (E) Distribution of risk scores stratified by grade. (F) Distribution of risk scores stratified by immune score. (G) Distribution of risk scores stratified by $M$ stage. $(\mathrm{H})$ Distribution of risk scores stratified by $\mathrm{N}$ stage. (I) Distribution of risk scores stratified by stage. $(\mathrm{J})$ Distribution of risk scores stratified by T stage. (K)PD -L1 expression in low/high risk. 

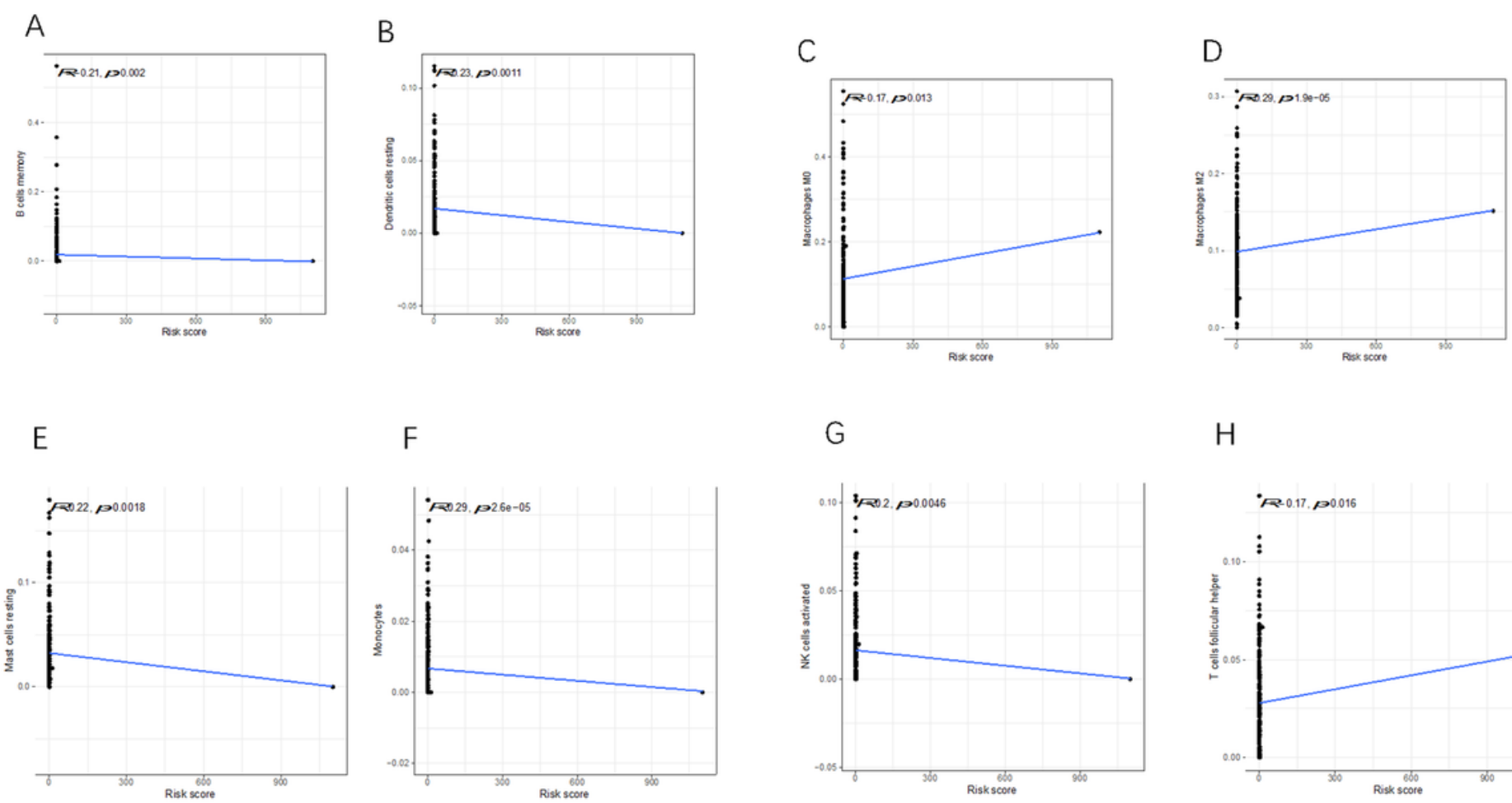

$\mathrm{F}$
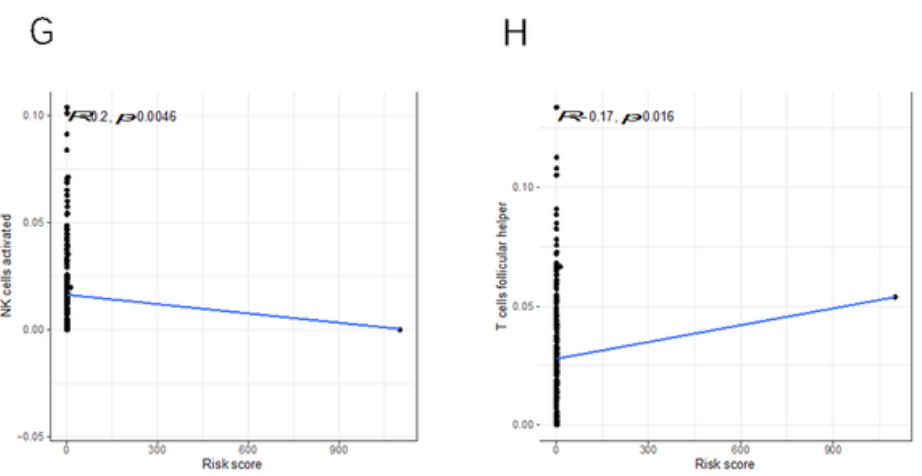

\section{Figure 7}

Relationship between the risk score and infiltration level of eight immune cell types. (A)B cells memory(B)Dendritic cells resting. (C) Macrophage M0. (D)Macrophages M2. (E)Mast cells resting. (F)Monocytes. (G)NK cells activated. $(H) T$ cells follicular helper. 